\title{
Electropolymerisation and properties of conducting polymers derived from aminobenzenesulphonic acids and from mixtures with aniline
}

\author{
Carolin Thiemann, Christopher M.A. Brett* \\ Departamento de Quimica, Universidade de Coimbra, 3004-535 Coimbra, Portugal
}

Received 19 March 2001; received in revised form 31 July 2001; accepted 3 August 2001

\begin{abstract}
The electrochemical polymerisation of 2- and 4-aminobenzenesulphonic acids (orthanilic acid and sulphanilic acid) in sulphuric acid solution and co-polymerisation of mixtures of aniline and acid monomers, have been systematically investigated. The polymers formed have been characterised voltammetrically. Further evidence concerning the polymer structure and chemical composition has been obtained by IR spectroscopy and scanning electron microscopy (SEM). C 2001 Elsevier Science B.V. All rights reserved.
\end{abstract}

Keywords: Aminobenzenesulphonic acids; Polyaniline; Self-doped polyaniline; Electrosynthesis; Electropolymerisation

\section{Introduction}

The polymerisation of substituted anilines can lead to interesting alterations in the conducting and other properties of polyaniline (PANI), see [1] for a general summary. This polymerisation can be carried out by chemical or electrochemical means, usually in strong acid solution. The introduction of substituents with an acidic group alters the acidity constants and can lead to a lesser drop in conductivity with increasing $\mathrm{pH}$. This modification of the polymer structure is referred to as "self-doping" in order to distinguish it from "external doping", which is due to the introduction of different counter-ions which can also influence the properties of the film, e.g. [2,3]. Acidic groups which can be used as aniline ring substituents are phosphonic [4], carboxylic $[1,5,6]$ and sulphonic [5,7-11]. Chemical polymerisation of $o$-aminobenzyl-phosphonic acid in aqueous solution gave poly (o-aminobenzylphosphonic acid) as a soluble functionalised polyaniline with a conductivity independent of $\mathrm{pH}$ in the range 0-6 [4]. A previous paper [1] has investigated the electropolymerisation of 2-, 3-, and 4-aminobenzoic acids, and summarised previous work carried out to synthesise various types of self-doped polyanilines. Sulphonic substituents have been introduced by sulphonation of polyaniline with fuming sulphuric acid, leading to partial substitution in the $o$-position [7-9]. The other route is by polymerising sulphonate-substituted anilines [10,11]. Although successful

\footnotetext{
* Corresponding author. Fax: +351-239-835295.

E-mail address: brett@ci.uc.pt (C.M.A. Brett).
}

chemical polymerisation has been reported, e.g. [12] and references therein, attempts to carry out electrochemical polymerisation of aminobenzenesulphonic acids by themselves have not been reported.

Co-polymerisation of aniline and substituted anilines can lead to polymers with intermediate properties. A number of investigations have been carried out with substituted anilines containing one or two sulphonic- substituents [13-20], but the influence of reaction conditions and the nature of the monomer on the polymerisation still need further examination.

In this paper, thin films of polymers derived from 2- and 4-aminobenzenesulphonic acids are prepared on glassy carbon electrode substrates by electrosynthesis and characterised electrochemically and spectroscopically, as well as by microscopic examination. Electrochemical co-polymerisation of aniline and aminobenzenesulphonic acid monomers has also been carried out in a similar way to that done with aminobenzoic acids [1], varying the ratio of the two monomers.

\section{Experimental}

2-aminobenzenesulphonic acid (Aldrich), 4-aminobenzenesulphonic acid (Aldrich), sulphuric acid (Riedel-deHaen) and sodium sulphate were used as received. Aniline (Riedel-de-Haen) was distilled under a nitrogen atmosphere at reduced pressure, kept in the dark, and used within 3 days. The electrolyte was usually 0.5 or $1.0 \mathrm{M}$ sulphuric acid, in some cases with the addition of sodium sulphate, for further 
details see [1]. Tridistilled water was used to make all solutions. Experiments were carried out at $25 \pm 1{ }^{\circ} \mathrm{C}$.

The electrochemical cell contained a glassy carbon disc working electrode (GCE), diameter $0.5 \mathrm{~cm}$, a platinum foil auxiliary electrode in a separate compartment connected by a glass frit and a Radiometer K401 saturated calomel electrode (SCE) as reference. The working electrode was polished with diamond lapping compound (Kemet International Ltd.) down to $1 \mu \mathrm{m}$ particle size on a polishing cloth. It was then held at a potential of $+1.6 \mathrm{~V}$ versus SCE in sulphuric acid electrolyte for $6 \mathrm{~min}$ and subsequently cycled at a sweep rate of $50 \mathrm{mV} \mathrm{s}^{-1}$ from -0.5 to $+1.5 \mathrm{~V}$ continuously for $15 \mathrm{~min}$. This treatment led to a reproducible voltammogram with much-reduced background currents.

Electrochemical polymerisation was carried out by cyclic potential sweep in sulphuric acid electrolyte solutions containing the monomer between potential limits as specified below, using an analogue potentiostat constructed in this laboratory. For polymerisation of the aminobenzenesulphonic acids alone a $0.05 \mathrm{M}$ solution was used, the solutions used for co-polymerisation experiments were 1:1-, 1:2or 2:1-mixtures of the aminobenzenesulphonic acid with aniline of total concentration $0.1 \mathrm{M}$.

Coated electrodes were transferred to a solution of pure electrolyte for electrochemical characterisation using the same potentiostat.

For electrochromism experiments, the films were deposited on a optically transparent electrode (OTE) (indium tin dioxide on a glass substrate of area $4 \mathrm{~cm}^{2}$ - Phosphor Products Ltd.) using the same arrangement. The OTE was wiped with ethanol and washed with distilled water before use.

Scanning electron microscopy (SEM) was performed on films deposited on OTE using a JEOL T330 microscope fitted with a Tracor Northern Microanalysis Accessory. Electrodes were sputter-coated with a thin gold film before SEM was undertaken (magnification $7500 \times$ at $20 \mathrm{kV}$ ).

For IR measurements pellets were pressed using $\mathrm{KBr}$ and FTIR spectra were obtained on Mattson Genesis instruments. Since the amount of film produced during one experiment for electropolymerisation at a scan rate of $50 \mathrm{mV} \mathrm{s}^{-1}$ was not sufficient, three films were prepared under the same conditions from the same solution and collected in a small glass tube. To remove any adherent monomers, the coated electrode was cycled three times in $1 \mathrm{M} \mathrm{H}_{2} \mathrm{SO}_{4}$, then the resulting precipitate was washed several times with ethanol to remove excess sulphuric acid, and dried.

\section{Results and discussion}

\subsection{Electrosynthesis}

Polymer films were obtained by potential cycling from both 2-aminobenzenesulphonic acid (2-ABS) and 4-aminobenzenesulphonic acid (4-ABS) when oxidised in sulphuric acid at a glassy carbon electrode. This result is in agreement with observations in [15] concerning the electropolymerisation of 2- and 3-ABS at a platinum electrode. It shows that by varying the reaction conditions (e.g. electrode material, electrolyte and positive potential limit), these substituted anilines can be electropolymerised, even though they are deactivated from both a steric and electronic point of view. For a general discussion of electronic and steric effects caused by electron-withdrawing groups on the polymerisation, see [1].

The electropolymerisation of 2-ABS was accompanied by brown streaks growing from the surface of the electrode into the solution. This "precipitate" was soluble in the electrolyte, even in neutral and also in basic solution. The films formed on glassy carbon were thin and powdery and were a yellow colour at $0 \mathrm{~V}$. A similar behaviour was observed with 4-ABS, with crimson streaking. Although some authors $[21,22]$ reported green soluble species as polyaniline side products, no such side products were observed here.

The anodic oxidation of mixtures of aminobenzenesulphonic acids and aniline yielded polymer films with good adhesion to the electrode substrate. These films were isolated at $0 \mathrm{~V}$ as green powdery substances. The addition of aniline reduced the formation of coloured streaks during electropolymerisation. During electrodeposition on ITO no streaks were observed.

\subsection{Cyclic voltammetry}

Fig. 1 shows cyclic voltammograms following the anodic oxidation of 2- and 4-ABS, at glassy carbon electrodes in sulphuric acid $\left(v=50 \mathrm{mV} \mathrm{s}^{-1}\right)$ after 30 cycles. The positive limit $(1.0 \mathrm{~V})$ was not lowered after the initiation period. Anodic current maxima occur at ca. $0.30 \mathrm{~V}$ (small peak), 0.49 and $0.66 \mathrm{~V}$ for $2-\mathrm{ABS}$ and at 0.39 and $0.48 \mathrm{~V}$ for 4 ABS. For the first 5 cycles the current of all peaks increased with each cycle, while after the 9th cycle the current of the peak at $0.49 \mathrm{~V}$ diminished steadily reaching about half of its maximum value by the 30th cycle. For all other peaks the currents increase, indicating progressive polymerisation and film formation.

This behaviour of 2- and 4-ABS contrasts with the observations made during the electropolymerisation of 2-, 3- and 4-aminobenzoic acids, where a steady increase of all peak currents was registered [1].

Table 1 gives data concerning peak currents from the $\mathrm{CV}$ registered for polymer films from 2- and 4-ABS alone and from mixtures of 2- and 4-ABS with aniline (Fig. 2). The position of the first anodic peak at $\sim 0.4 \mathrm{~V}$ versus SCE is the same in polymerisation mixtures as for aniline. Compared to aniline, the rate of film growth as expressed by the peak current, is higher in mixtures, particularly those that contain less aniline (1:2 ratio) for both 2- and 4-sulphonic acid substituents. This behaviour is completely different to that with carboxylic acid substituents where an equal molar ratio of aniline was necessary for reasonable film growth. The directing power of the carboxylic and sulphonic acid 

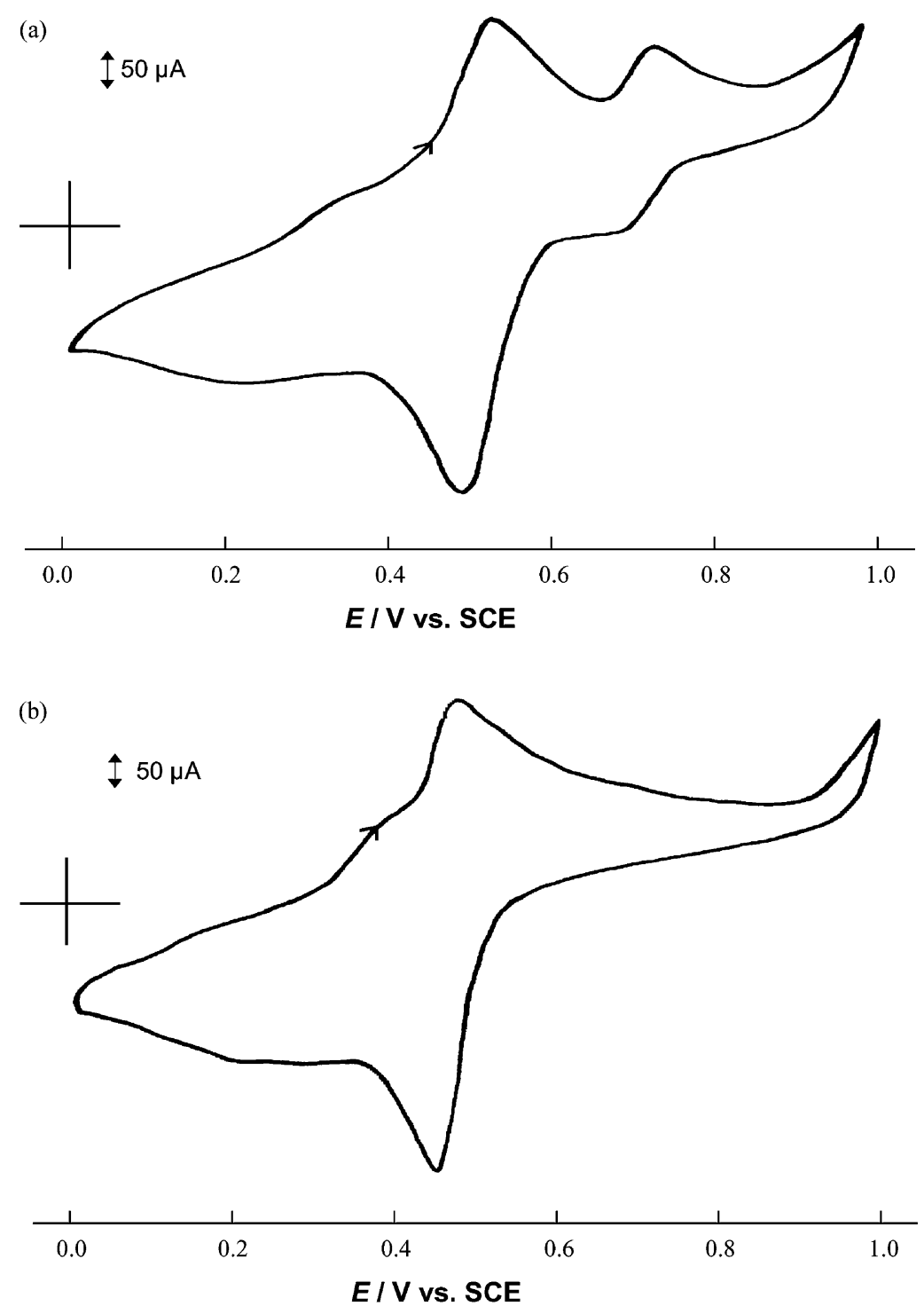

Fig. 1. Cyclic voltammograms of polymer films formed in $1.0 \mathrm{M} \mathrm{H}_{2} \mathrm{SO}_{4}$ on glassy carbon electrodes from: (a) 2-aminobenzenesulphonic acid; (b) 4aminobenzenesulphonic acid.

substituents is the same ( $m$-directing). Therefore, the explanation must be linked to a preferential interaction between the growing polyaniline chains with the substituted monomer promoting further growth and there may be an effect on chain branching. This phenomenon could be further

\section{Table 1}

Peak currents $(\mathrm{mA})$ at glassy carbon electrode for the first anodic peak for polyaniline and co-polymers from anilines with aminobenzenesulphonic acids (ABS), for different monomer ratios ${ }^{\mathrm{a}}$

\begin{tabular}{lllll}
\hline & $\begin{array}{l}\text { Aniline or } \\
\text { ABS alone }\end{array}$ & \multicolumn{4}{l}{ Aniline/ABS ratio } \\
\cline { 3 - 5 } & & $2: 1$ & $1: 1$ & $1: 2$ \\
\hline Aniline & 2.25 & - & - & - \\
2-ABS & 0.029 & 2.63 & 2.95 & 3.95 \\
4-ABS & 0.031 & 2.90 & 2.80 & 4.05 \\
\hline
\end{tabular}

${ }^{\mathrm{a}}$ Positive potential limit $1.0 \mathrm{~V} ; 30$ cycles at $50 \mathrm{mV} \mathrm{s}^{-1}$. explored in future studies. For a general discussion of the polymerisation mechanism, see [1]. During co-polymerisation of 4-ABS and aniline anodic oxidation peaks tended to shift to higher potentials with a rising number of cycles, reflecting an increasing contribution from the resistance of the films. This phenomenon was otherwise only found for polymerisations at a scan rate of $10 \mathrm{mV} \mathrm{s}^{-1}$ and might be due to a larger amount of film formed in these experiments.

\subsection{Chromatic properties}

The visual impression of the as-formed films was noted. Except for the colour at the potential of $-0.2 \mathrm{~V}$, all observations were made during electrodeposition of the film on the indium tin oxide (ITO) conducting glass surface.

The polymers formed from 2- and 4-ABS did not have any colour for any value of applied potential. The films formed 

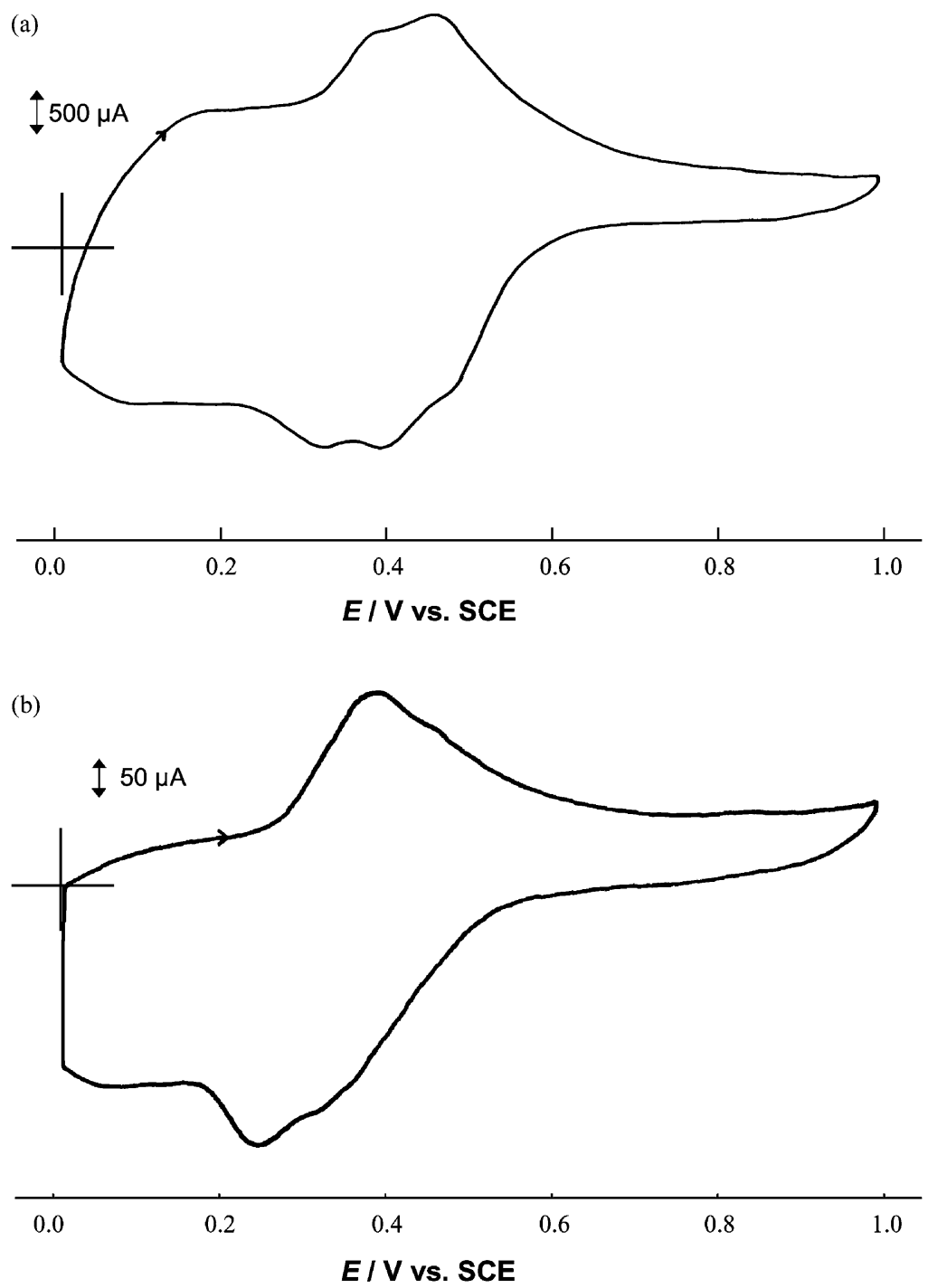

Fig. 2. Cyclic voltammograms of polymer films formed in $1.0 \mathrm{M} \mathrm{H}_{2} \mathrm{SO}_{4}$ on glassy carbon electrodes from 1:1 mixtures of aniline and: (a) 2aminobenzenesulphonic acid; (b) 4-amino-benzenesulphonic acid.

from ABS/aniline mixtures demonstrated the same change of colour from light yellowish green $(-0.2 \mathrm{~V})$ to dark blue $(+0.9 \mathrm{~V})$, as already observed for polymer films from aminobenzoic acids with aniline [1] and from polyaniline [22]. The colours intensified with film thickness.

This electrochromic behaviour from -0.2 to $0.9 \mathrm{~V}$ was reproducible and stable with time with a gradual change in colour, whereas for films from polyaniline the change of colour with applied potential is usually much sharper [23]. This could make the films from monomer mixtures interesting materials for application in certain types of electrochromic device, where a damped response to an external perturbation is required.

\subsection{Morphology}

Fig. 3a shows an SEM micrograph of a typical area of the deposit of poly(2-ABS) formed in $1 \mathrm{M}$ sulphuric acid.
The film has a grainy structure with leaf-like growths. No dot-like structures attributed to residual $\mathrm{H}_{2} \mathrm{SO}_{4}$ were found [1]. Although the cracks probably derive from the drying of the film, similar cracks were found in films of poly(o-toluidine) synthesised at temperatures above $35^{\circ} \mathrm{C}$ [24]. There was not sufficient deposit of poly(4-ABS) to make it possible to distinguish features from the background film, reflecting the slower growth from the 4-ABS monomer.

All SEM micrographs of films derived from co-polymerisations of 2- and 4-ABS with aniline show crystalline formations on top of a background film. The background film (for example Fig. 3b) resembles the texture found for polyaniline formed at a slow scan rate [25]. The crystalline structures occur in two different shapes: some are rod-like (Fig. 3c), while others are disc-like and form larger complexes (Fig. 3d); both of these areas can be found in the same film. Although films derived from co-polymerisations of 


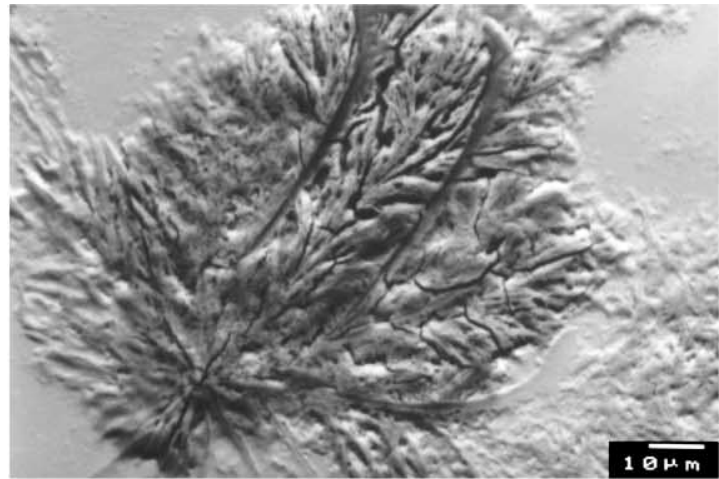

(a)

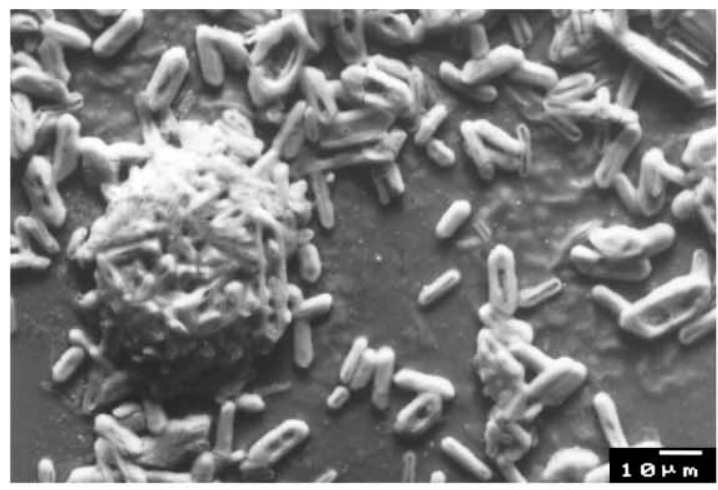

(c)

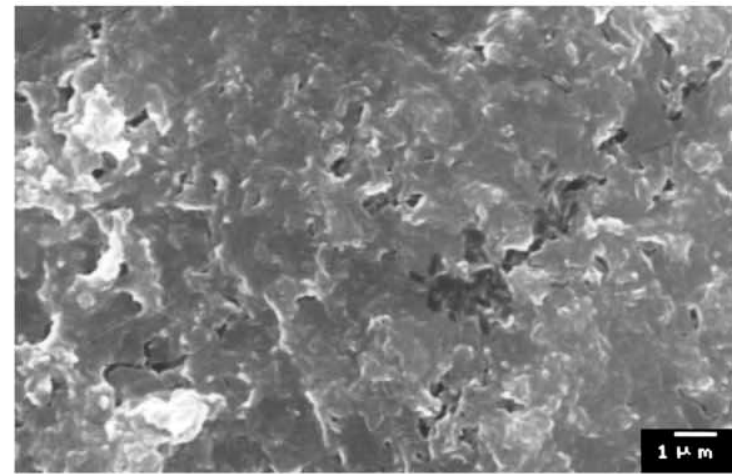

(b)

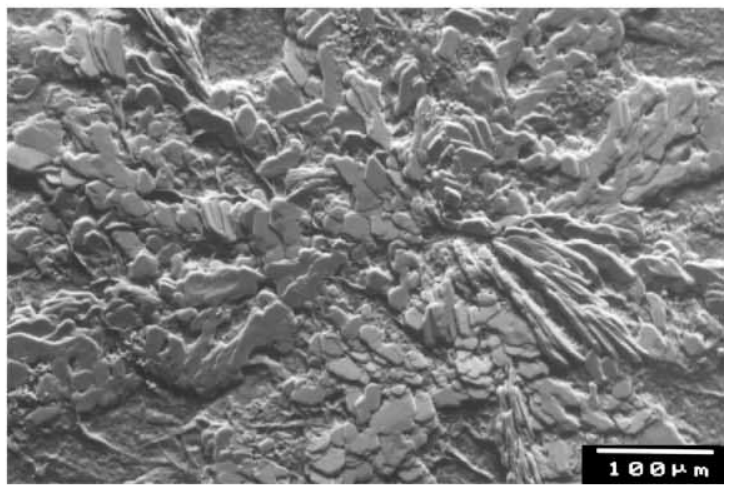

(d)

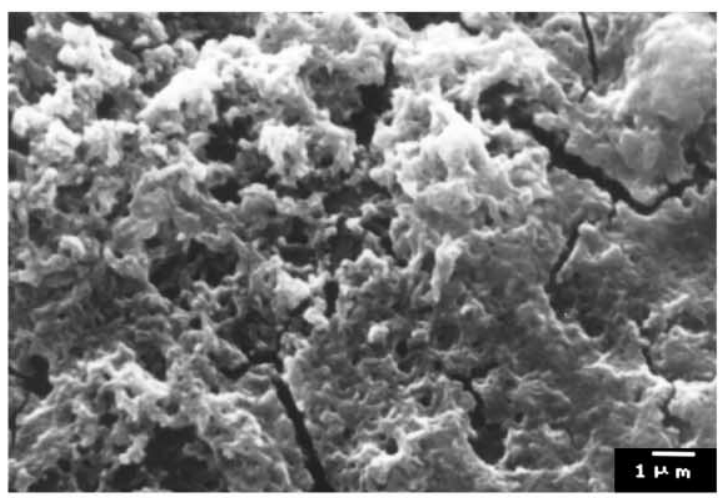

(e)

Fig. 3. Scanning electron micrographs of polymeric films formed on ITO electrodes in sulphuric acid solution (30 cycles at $50 \mathrm{mV} \mathrm{s}^{-1}$ scan rate between 0.0 and 1.0 V) from: (a) 2-aminobenzenesulphonic acid; (b) 4-aminobenzenesulphonic acid/aniline 2:1; (c) 2-aminobenzenesulphonic acid/aniline 1:1; (d) 4aminobenzenesulphonic acid/aniline 1:1 (e) 4-aminobenzenesulphonic acid/aniline $1: 1$, scan rate $10 \mathrm{mV} \mathrm{s}^{-1}$ during polymerisation.

aminobenzoic acids with aniline under the same conditions also have a kind of duplex texture, these films contain no features similar to the above-mentioned structures.

While the textures of polymers formed from 1:1 mixtures of 2- or 4-ABS and aniline at a scan rate of $50 \mathrm{mV} \mathrm{s}^{-1}$ contain crystalline elements and a background film (Fig. 3d), films synthesised at a scan rate of $10 \mathrm{mV} \mathrm{s}^{-1}$ show a more uniform, porous texture, Fig. 3e. This texture is slightly less porous that found for films resulting from co-polymerisation of aminobenzoic acids with aniline under the same conditions.

\subsection{IR spectroscopy}

Fig. 4 shows the IR spectra of the polymer films formed at glassy carbon by anodic oxidation of 1:1 mixtures of ABS, Fig. $4 \mathrm{a}-\mathrm{c}$, and aniline, Fig. $4 \mathrm{~d}$.

Polymers from 2- and 4-ABS both show relatively strong absorption peaks at $884(865)$ and $850 \mathrm{~cm}^{-1}$, which arise from the out-of-plane bending of a $\mathrm{C}-\mathrm{H}$ bond [26], while polyaniline itself has only one band in this region, as expected for a 1,4 di-substituted aromatic compound [27,28]. As occurs with polymers formed from aminobenzoic acids, 

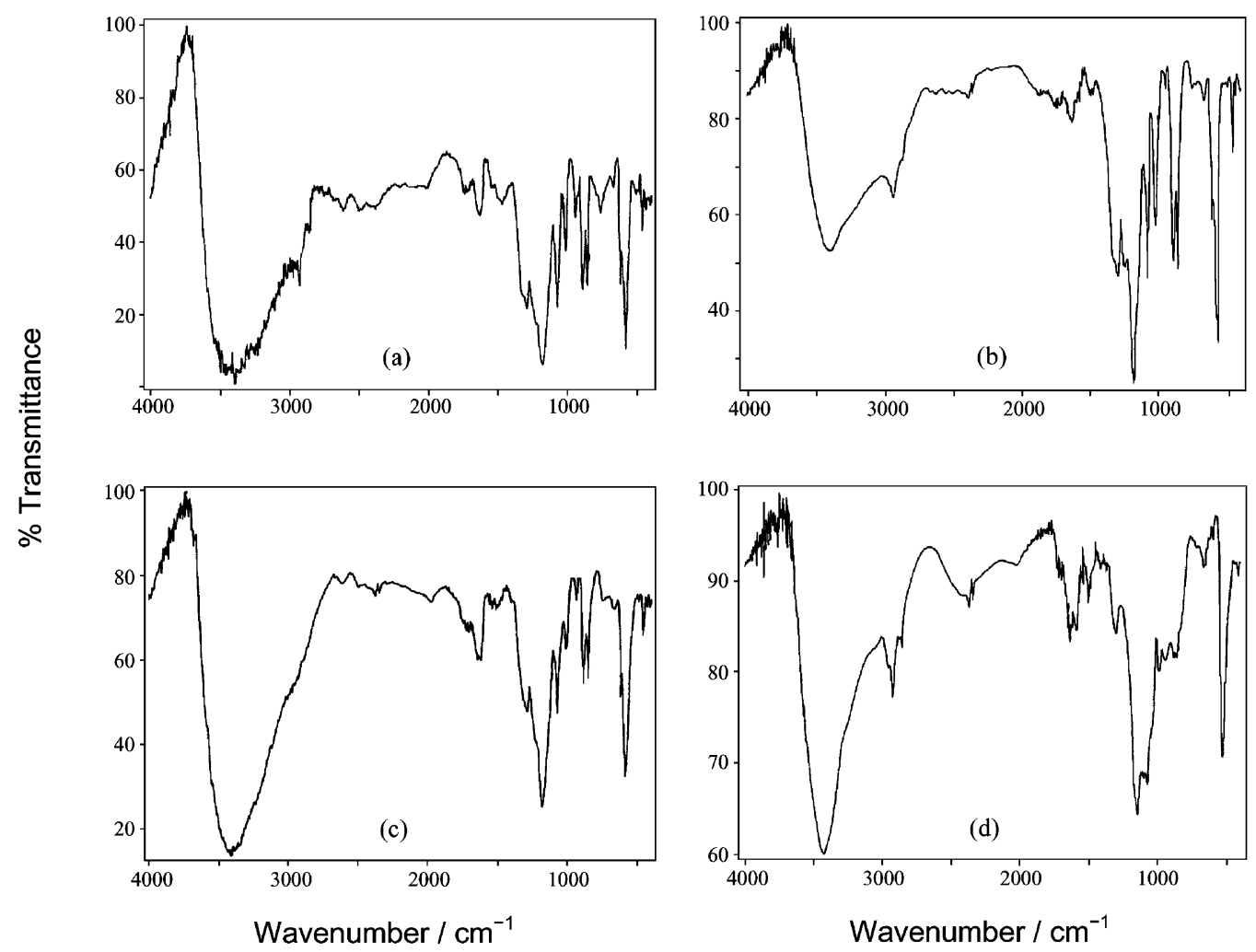

Fig. 4. Infrared spectra of polymer films formed in $1.0 \mathrm{M} \mathrm{H}_{2} \mathrm{SO}_{4}$ at glassy carbon electrodes from (a) 1:1 mixture of aniline and 2-aminobenzenesulphonic acid; (b) 1:1 mixture of aniline and 4-aminobenzenesulphonic acid, scan rate $50 \mathrm{mV} \mathrm{s}^{-1}$; (c) 1:1 mixture of aniline and 4-aminobenzenesulphonic acid, scan rate $10 \mathrm{mV} \mathrm{s}^{-1}$ (d) aniline.

the films, therefore, probably contain branched structures derived from a coupling in 1-, 3- and 5- or 1-, 2- and 4position of the aromatic ring as well as linear elements derived from coupling in the 1- and 4-positions [29].

Peaks in the range $3500-3300 \mathrm{~cm}^{-1}$ correspond to the stretching of $\mathrm{N}-\mathrm{H}$ bonds, which appear in these spectra at ca $3400 \mathrm{~cm}^{-1}$ for both polymers, while weaker peaks between 2700 and $2250 \mathrm{~cm}^{-1}$ suggest the existence of ammonium salts. The IR spectra of the polymers from both 2- and 4ABS have several peaks in that region.

Peaks in the region between 1690 and $1490 \mathrm{~cm}^{-1}$ are due to the deformation of different types of $\mathrm{N}-\mathrm{H}$ bond. Absorption $>1600 \mathrm{~cm}^{-1}$ can be attributed to carbon-nitrogen double bonds, while weak absorption between 1580 and $1490 \mathrm{~cm}^{-1}$ is typical for secondary amines. All IR spectra of the mixtures show some absorption in the latter region, which seems feasible since all films were collected at $0 \mathrm{~V}$ in the reduced state of the polymer.

Bands at 1174 and $613 \mathrm{~cm}^{-1}$ derive from the presence of sulphate ions in the film [30], which have a high intensity since the polymerisation was carried out in sulphuric acid. It is difficult to estimate the extent to which sulphonic groups incorporated in the polymer contribute to the bands found in the spectra, since the position and number of the characteristic bands for benzenesulphonic acids [29,30] changes from monomer to polymer and may overlap those typically found for sulphate anions. This is demonstrated by the fact that
$v\left(\mathrm{SO}_{3^{-}}\right)$is at 1113 and $617 \mathrm{~cm}^{-1}$ for 4,4-diaminodiphenylamine sulphate [31] and $v\left(\mathrm{SO}_{3}-\right)$ is at $1230,1206,1064$, 1027 and $558 \mathrm{~cm}^{-1}$ for 2-aminobenzenesulphonic acid and at $1157,1124,1034,682$ and $558 \mathrm{~cm}^{-1}$ for 4-aminobenzenesulphonic acid [32].

Variation of the scan rate during electrosynthesis clearly alters film characteristics such as morphology, see above, and can be monitored by cyclic voltammetry. However, such differences have very little influence on the shape of the IR spectrum in the region $<1500 \mathrm{~cm}^{-1}$, although the broad band between 3000 and $3500 \mathrm{~cm}^{-1}$ does increase in size and becomes closer to that of polyaniline (see Fig. $4 \mathrm{~b}$ and c: 4-ABS/aniline $1: 1, v=50$ and $10 \mathrm{mV} \mathrm{s}^{-1}$ ).

\subsection{Comparative remarks}

The results shown above have demonstrated the lower rate of polymerisation of sulphonate-substituted anilines compared to aniline and that the polymerisation rate of 4-ABS is similar to that of 2-ABS. In particular there is evidence of branching, e.g. coupling in the 2- and 5-positions must have occurred during polymerisation. Since it is more difficult to happen due to steric reasons, at least in the initial stages, the influence of the substrate can be expected to be greater, as was observed in this work: whereas 4-ABS polymerises on glassy carbon, polymerisation on ITO electrodes is extremely slow. 
Thus, there can be formation of branched as well as of linear polymer chains. This also happens with aminobenzoic acids [1]. Therefore, the polymer structure reflects the substitution pattern of the monomer, i.e. electronic considerations have to be taken into account when proposing a polymerisation mechanism. Alternative coupling in the $o$ position to the amino-group would explain why the morphology of electrochemically grown polyaniline is often characteristic of a branched polymer, particularly if the polymerisation is rapid. Since there are two $o$-positions, the probability of coupling at an $o$-position is quite high, but becomes more difficult for longer chains. In these cases, degradation of the polymer is also faster. So in many cases, formation of only a linear polymer may not correspond to reality. Such a conclusion is borne out by the exceptional stability of polyaniline prepared by chemical polymerisation at low temperature where high linearity is achieved.

\section{Conclusion}

The anodic oxidation of both 2-aminobenzenesulphonic acid and 4-aminobenzenesulphonic acid yielded conducting polymers. This demonstrates that it is not essential for a successful polymerisation to have no substituent in the $p$ position to the amino group. Although 4-ABS formed a conducting polymer film on glassy carbon, it showed little inclination to form a deposit on ITO, showing the importance of the substrate in the initial nucleation step. Further characterisation (impedance measurements) and variation of behaviour with $\mathrm{pH}$ for these polymers will be presented elsewhere [33].

When aniline was used as co-monomer, the resulting films showed properties characteristic of both poly(aminobenzenesulphonic acid)s and polyaniline. The composition of the polymeric films did not reflect the ratio of the monomers during electropolymerisation, as the aminobenzenesulphonic acids are less easily oxidised than aniline. The rate of film growth is low even for mixtures containing a large amount of aniline monomer. This means that the polymerisation rate depends on the rate of oxidation of the aminobenzenesulphonic acid monomer, and reflects the rate-determining role of the aminobenzenesulphonic acid monomers in polymer formation.

\section{References}

[1] C. Thiemann, C.M.A. Brett, Synth. Met. 123 (2001) 1.

[2] C.M.A. Brett, A.M. Oliveira Brett, J.L.C. Pereira, C. Rebelo, J. Appl. Electrochem. 23 (1993) 332.

[3] M. Karakisla, M. Sacak, E. Erdem, U. Akbulut, J. Appl. Electrochem. 27 (1997) 309

[4] S.C. Ng, H.S.O. Chan, H.H. Huang, P.K.H. Ho, J. Chem. Soc., Chem. Commun. (1995) 1327.

[5] A.A. Karyakin, A.K. Strakhova, A.K. Yatsimirsky, J. Electroanal. Chem. 371 (1994) 259.

[6] N. Toshima, H. Yan, Y. Gotoh, M. Ishiwatari, Chem. Lett. (1994) 2229.

[7] J. Yue, A.J. Epstein, A.G. MacDiarmid, Mol. Cryst. Liq. Cryst. 183 (1990) 255.

[8] X. Wei, A.J. Epstein, Synth. Met. 74 (1995) 123.

[9] X.-H. Wang, J. Li, L.-X. Wang, X.-B. Jing, F.-S. Wang, Synth. Met. 69 (1995) 147.

[10] A.A. Karyakin, I.A. Maltsev, L.V. Lukachova, J. Electroanal. Chem. 402 (1996) 217.

[11] S. Shimizu, T. Saitoh, M. Uzawa, M. Yuasa, K. Yano, T. Maruyama, K. Watanabe, Synth. Met. 85 (1997) 1337.

[12] B.C. Roy, M.D. Gupta, L. Bhowmik, J.K. Ray, Synth. Met. 100 (1999) 233.

[13] J.Y. Lee, C.Q. Cui, X.H. Su, M.S. Zhou, J. Electroanal. Chem. 360 (1993) 177.

[14] J.Y. Lee, C.Q. Cui, J. Electroanal. Chem. 403 (1996) 109.

[15] M. Pasquali, G. Pistoia, R. Rosati, Synth. Met. 58 (1993) 1.

[16] C. Barbero, R. Kötz, Adv. Mat. 6 (1994) 577.

[17] P.A. Kilmartin, G.A. Wright, Synth. Met. 88 (1997) 153.

[18] P.A. Kilmartin, G.A. Wright, Synth. Met. 88 (1997) 163.

[19] H. Tang, A. Kitani, S. Ito, Electrochim. Acta. 42 (1997) 3421.

[20] H. Tang, A. Kitani, T. Yamashita, S. Ito, Synth. Met. 96 (1998) 43.

[21] D. Bloor, A. Monkman, Synth. Met. 21 (1987) 175.

[22] C.D. Batich, H.A. Laitinen, H.C. Zhou, J. Electrochem. Soc. 137 (1990) 883.

[23] T. Kobayashi, H. Yoneyama, H. Tamura, J. Electroanal. Chem. 161 (1984) 419

[24] P. Ocon, P. Herrasti, New J. Chem. 16 (1992) 501.

[25] L.H. Dao, M. Leclerc, J. Guay, J.W. Chevalier, Synth. Met. 29 (1989) E377.

[26] M. Hesse, H. Meier, B. Zeeh, Spektroskopische Methoden in der Organischen Chemie, G. Thieme Verlag, Stuttgart, 1979.

[27] M.A. Habib, S.P. Maheswari, J. Electrochem. Soc. 136 (1989) 1050.

[28] L. Duic, Z. Mandic, J. Electroanal. Chem. 335 (1992) 207.

[29] E.M. Genies, M. Lapkowski, J.F. Penneau, J. Electroanal. Chem. 249 (1988) 97.

[30] T. Ohsaka, Y. Ohnuki, N. Oyama, J. Electroanal. Chem. 161 (1984) 399.

[31] K.G.R. Pachler, F. Matlock, H.-U. Gremlich, Merck Ft-IR Atlas, VCH Verlagsgesellschaft mbH, Weinheim, 1988.

[32] L. Ganguly, C.I. Jose, A.B. Biswas, Spectrochim. Acta. Part A 24 (1968) 215.

[33] C. Thiemann, C.M.A. Brett, in preparation. 